\title{
Elemente und Ephemeriden der Planeten (291) und (292).*)
}

Die Elemente, dic ich hier mitzutheilen mir erlaube, zeigen, dass beide Planeten vom 25. April neu sind. Für (291) hatte ich erst ein Elementenșystem aus 1890 April 25 und Mai ro Wien und Mai I 6 Nizza abgeleitet; dasselbe schien aber noch unsicher bestimmt, und so wiederholte ich die Rechnung, nachdem heute noch Wiener Beobachtungen von Herrn Dr. Palisa am 18. und 19. Mai eingetroffen waren. Aus April 25, Mai so und I9 hat sich ergeben :

$$
\begin{aligned}
& \text { Epoche I } 890 \text { Mai i } 9.5 \text { M. Z. Berlin } \\
& M=85^{\circ} 2 \mathbf{I}^{\prime} 8^{\prime \prime} .5 \\
& \omega=324 \quad 28 \quad 18.5 \\
& \delta=\begin{array}{lll}
161 & 25 & 26.1 \\
\beta & \text { M. Aequ. } 1890.0
\end{array} \\
& i=15152.0 \\
& \varphi=6348.2 \\
& \mu=1065^{\prime \prime} 59 \mathrm{I}
\end{aligned}
$$$$
\log a=0.348277
$$

Dieser Planet stand im Oct. I 875 etwa 45 Grad (hel.) von dem Orte des Planeten (149) Medusa ab.
Für den anderen Planeten, (292), fand ich aus April 25 und Mai 9 Wien, und Mai 6 Nizza:

$$
\begin{aligned}
& \text { Epoche } 1890 \text { Mai I6.5 M. Z. Berlin } \\
& M=268^{\circ} 21^{\prime} 23^{\prime \prime} 8 \\
& \omega=274 \quad 9 \text { r } 0.3 \\
& \delta=\begin{array}{lll}
43 & 8 & \text { r } 2.4
\end{array} \text { M. Aequ. } 1890.0 \\
& i=\begin{array}{lll}
444 & 4.4
\end{array} \\
& \varphi=22521.9 \\
& \mu=879.354 \text {. } \\
& \log a=0.403895
\end{aligned}
$$

\begin{tabular}{|c|c|c|c|c|c|c|c|}
\hline \multicolumn{2}{|c|}{1890} & $\alpha$ & & $\delta$ & & $\log r$ & $\log 4$ \\
\hline Juni & 3 & I $3^{\mathrm{h}} 53^{\mathrm{m}} 54^{\mathrm{s}}$ & - & $8^{\circ}$ & $46: 2$ & 0.3529 & 0.1488 \\
\hline & 5 & 5334 & & & $45 \cdot 7$ & & \\
\hline & 7 & 5320 & & & 45.9 & $0.353^{8}$ & 0.1604 \\
\hline & 9 & 53 I 4 & & 8 & 46.8 & & \\
\hline & 11 & $53 \quad 14$ & & 8 & 48.3 & 0.3547 & 0.1723 \\
\hline & 13 & 5322 & & & 50.5 & & \\
\hline & I 5 & 5337 & & 8 & 53.4 & $0.355^{6}$ & 0.1844 \\
\hline & 17 & $535^{8}$ & & 8 & $5^{6.9}$ & & \\
\hline & 19 & 5426 & & 9 & I. I & $0.35^{65}$ & 0.1968 \\
\hline & 21 & 550 ? & & 9 & $5 \cdot 9$ & & \\
\hline & 23 & 135541 & $\longrightarrow$ & 9 & I I $\cdot 3$ & 0.3574 & 0.2093 \\
\hline
\end{tabular}

\begin{tabular}{|c|c|c|c|c|c|c|c|c|}
\hline \multicolumn{2}{|c|}{1890} & & $\alpha$ & & \multicolumn{2}{|c|}{$\delta$} & $\log r$ & $\log \Delta$ \\
\hline Juni & 3 & 13 & $46^{n}$ & $9^{\text {s }}$ & $-11^{\circ}$ & $48: 2$ & 0.4038 & $0.23^{2} 3$ \\
\hline & 5 & & 45 & 22 & I I & 55.0 & & \\
\hline & 7 & & 44 & 4 I & 12 & $2 . I$ & 0.4035 & $0.241 \mathrm{I}$ \\
\hline & 9 & & 44 & 7 & I 2 & 9.6 & & \\
\hline & I 1 & & 43 & 40 & I 2 & 17.5 & $0.403^{2}$ & 0.2503 \\
\hline & I 3 & & 43 & 20 & 12 & 25.8 & & \\
\hline & I 5 & & 43 & 6 & 12 & 34.5 & 0.4028 & 0.2599 \\
\hline & I 7 & & 42 & 59 & I 2 & $43 \cdot 5$ & & \\
\hline & I 9 & & 42 & 59 & 12 & 52.9 & 0.4025 & 0.2697 \\
\hline & 2 I & & 43 & 5 & I 3 & 2.7 & & \\
\hline & 23 & 13 & 43 & I 8 & -13 & I 2.8 & 0.4022 & $0.279^{6}$ \\
\hline
\end{tabular}

Eine Beobachtung von Wien Mai 19 stimmt noch genügend, so dass die Elemente nicht mehr sehr irrig sein können.

Alsdann war aber der Planet (292) sowohl im Jahre 1868 von Dike wie 1875 von Scylla $z u$ weit entfernt, als dass er mit einem derselben identisch sein könnte.

Aus den obigen Elementen folgen die nachstehenden Ephemeriden für $12^{\mathrm{h}} \mathrm{M}$. Z. Berlin : Planet (291). Gr. $13^{\mathrm{m}}$.

Berlin 1890 Mai 26.
Planet (292). Gr. I $2^{\mathrm{m}}$.

A. Berberich.

*) Nachdem nunmehr die in den letzten Monaten entdeckten Planeten sich sämmtlich als neu herausgestellt haben, ist die Numerirung folgendermassen festzustellen:

(289) Planet Charlois 189o März 10

(290) , Palisa 1890 März 20
(291) I. Planet Palisa 189o April 25

(292) II. Planet Palisa I 890 April 25

$K r$.

Entdeckung eines neuen Planeten (293) auf der Sternwarte in Nizza.

Am 2 I. Mai Nachm. erhielt die Centralstelle folgendes Telegramm von Herrn Perrotin:

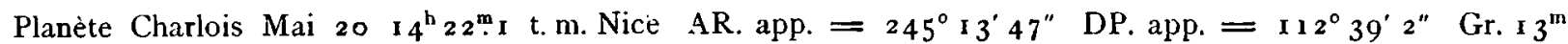

Das Telegramm wurde sofort weiter befördert.

\section{Inhalt:}

Zu $\mathrm{Nr}$, 2969. 7. Scheiner. Ueber die Bestimmung von Sterngrössen aus photographischen Aufnahmen. 273. - F. Scheiner. Apparat zur Verbreiterung von photographischen Sternspectren, 279, - W. Luther. Bemerkungen zu einigen auf Hamburger Beobachtungen beruhenden Sternpositionen des Catalogs von Dr. Kam. 281. - B. von Engelhardt. Beobachtungen von Cometen. 283. - F. Bidschof. Ephemeride des Cometen I89o... (Brooks März I9). 285. - A. Berberich. Elemente und Ephemeriden der Planeten (291) und (292). 287. - Entdeckung eines neuen Planeten (293) auf der Sternwarte in Nizza. 287. 